\title{
Filigrane
}

Écoutes psychothérapiques

\section{Une analysante, traductrice de Freud, " traduit " dans la cure et l'écriture un héritage de survivants}

\section{Janine Altounian}

Volume 20, numéro 1, printemps 2011

La langue vivante de la clinique psychanalytique

URI : https://id.erudit.org/iderudit/1004036ar

DOI : https://doi.org/10.7202/1004036ar

Aller au sommaire du numéro

Éditeur(s)

Revue Santé mentale au Québec

ISSN

1192-1412 (imprimé)

1911-4656 (numérique)

Découvrir la revue

Citer cet article

Altounian, J. (2011). Une analysante, traductrice de Freud, « traduit » dans la cure et l'écriture un héritage de survivants. Filigrane, 20(1), 11-21.

https://doi.org/10.7202/1004036ar
Résumé de l'article

L'auteur se propose d'établir un parallèle, dans sa double activité d'essayiste et de cotraductrice de Freud depuis 1970, entre deux types de traduction : la traduction d'une langue à une autre et la traduction en mots - effectuée dans le champ transférentiel et dans celui de l'écriture - de ce qui n'en disposait pas pour se dire. Une certaine analogie peut ainsi s'établir entre la traduction linguistique et celle du travail analytique dans la cure ou l'écriture, soit parce que les mots de l'original sont étrangers à cet autre qu'est le lecteur, soit parce que l'originel, transmis sans mots, doit trouver ses mots en présence de l'autre du transfert. 


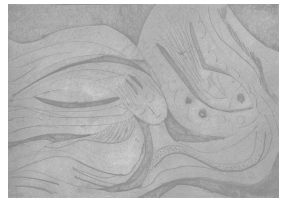

\title{
Une analysante, traductrice de Freud, "traduit " dans la cure et l'écriture un héritage de survivants
}

\author{
Janine Altounian
}

\begin{abstract}
L'auteur se propose d'établir un parallèle, dans sa double activité d'essayiste et de cotraductrice de Freud depuis 1970, entre deux types de traduction: Ia traduction d'une langue à une autre et la traduction en mots - effectuée dans le champ transférentiel et dans celui de l'écriture - de ce qui n'en disposait pas pour se dire. Une certaine analogie peut ainsi s'établir entre la traduction linguistique et celle du travail analytique dans la cure ou l'écriture, soit parce que les mots de l'original sont étrangers à cet autre qu'est le lecteur, soit parce que l'originel, transmis sans mots, doit trouver ses mots en présence de l'autre du transfert.
\end{abstract}

e présent article trouve un heureux appui dans un fait de langue en allemand: le même substantif « Übertragung» désigne à la fois «transfert » et «traduction ${ }^{1}$ ». Il se propose en effet de montrer comment c'est le «transfert ou traduction» qui promeut aussi bien la pratique langagière d'une héritière de survivants dans le cadre de la cure et de l'écriture que sa pratique de traductrice. L'argumentation qui suit se propose donc de répondre aux interrogations de collègues me demandant pourquoi je n'étais pas ou ne voulais pas être psychanalyste, malgré la publication d'ouvrages sur la transmission traumatique qui témoignaient d'une certaine expérience analytique (Altounian, 1990 ; 2000 ; 2005, 2009). Pensant que la curiosité apparemment biographique de mes interlocuteurs renvoyait à un questionnement de fond, j'avais d'abord répondu que mon écriture ne se référait pas à l'expérience clinique d'une psychanalyste mais à celle d'une analysante qui cherchait à «traduire » ce qui du silence des survivants aux meurtres de masse se transmettait à leurs enfants. J'aimerais néanmoins ajouter ici quelques explications sur les différents points de convergence d'un parcours où se sont conjugués, chez moi, deux formes d'expression divergentes : l'écriture d'une analysante et la traduction de Freud depuis 1970.

La première idée qui me vient pour introduire mon propos est de soutenir que, dans les postures à l'œuvre dans ces deux modes d'activités linguistiques, la «pulsion à traduire» naît d'une nécessité à défier paradoxalement l'impossibilité de la traduction, soit d'un plaisir, soit d'une perte. Le plaisir serait, dans la première activité, celui pris à la langue d'origine ignorée du lecteur étranger à cette langue et, dans la 
seconde, celui procuré par l'amour secret des ascendants vécu naguère dans le mutisme. Quant à la perte, elle serait, soit la perte de la polysémie des mots lors du passage d'une langue-source à la langue-cible, soit la douleur partagée inconsciemment dans l'enfance avec ceux qui avaient survécu à la perte de leur pays et de leur vie. Il s'agirait en somme, dans les deux situations, de chercher respectivement une voie de sublimation à l'inconfort angoissant de se trouver au croisement périlleux de deux modes de réception du sens ou des émotions, deux codes délivrant de part et d'autre les déterminants de la pensée et de la vie affective.

Ce parallèle, qu'il s'agit donc d'expliciter entre les processus à l'œuvre lors de la traduction d'une langue à une autre et lors de la traduction, en une langue, de ce qui ne pouvait s'exprimer en un langage, me fut suggéré pour la première fois à la journée sur L'impact des mots, organisée par Nathalie Zaltzman dont certaines observations sur mon livre L'écriture de Freud. Traversée traumatique et traduction, mettaient en évidence la thématique des restes dans mon travail (Topique, 96, 88-89) ${ }^{2}$. Celui-ci s'attache, il est vrai, à une prise en compte des restes et du déplacement, aussi bien dans le travail analytique que dans celui de la traduction. Traduire c'est bien, dans les deux cas de figure, déplacer de l'original vers l'autre langue, et de l'originel vers le transfert. Une certaine analogie peut ainsi s'établir entre la traduction linguistique et celle, effectuée dans le champ transférentiel, de ce qui ne dispose pas de mots pour se dire à l'autre, soit parce que les mots de l'original sont étrangers à cet autre, soit parce que l'originel, transmis sans mots, doit trouver ses mots en présence de l'autre. Quant à ce qui « reste» difficilement traduisible, soit le plaisir ou la douleur de teneur et de portée différentes dans l'une et l'autre des configurations, il constitue un point de jonction où se sont recoupés chez moi le travail d'une analysante face au matériel transmis par une famille de survivants et celui d'une traductrice face à un texte fondateur.

\section{Exemple de perte dans la traduction de Freud}

Voici donc un exemple de plaisir à la langue de Freud mais aussi de frustration pour le traducteur empêché de faire sentir au lecteur, sans passer par le commentaire qui va suivre, l'élégance d'un style mise au service d'une pensée. Je me bornerai ici à une illustration unique de la façon dont, chez Freud, la sensibilité de l'écrivain perce à même la langue du chercheur et rend alors impossible la traduction de cette «percée» métalinguistique:

Nous connaissons tous les dernières lignes d'Actuelles sur la guerre et la mort:

Nous nous souvenons du vieil adage: Si tu veux maintenir (erhalten willst) la paix, arme pour la guerre. Il serait d'actualité de le modifier: Si tu veux endurer (aushalten willst) la vie, organise-toi en vue de la mort. (Freud, OCF/P, 1988, 157; GW, $1975,355)^{3}$

Freud prend ici la liberté de diffracter le même syntagme latin «si vis (si tu veux)» en deux variations verbales en allemand selon que le complément d'objet en est «la paix» ou «la vie» : Pour le syntagme «Si vis pacem (si tu veux la paix)», il traduit «si 
vis» par «wenn du... erhalten willst (si tu veux maintenir...)», pour le syntagme «Si vis vitam (si tu veux la vie)», il traduit «si vis» par «wenn du... aushalten willst (si tu veux endurer...)». En passant ainsi de «erhalten (maintenir)» quand il s'agit de la paix à «aushalten (endurer)» quand il s'agit de la vie, il module le radical du verbe «halten (tenir)» en substituant la particule aus à la particule er. Ce simple remplacement d'une particule par une autre trahit, parallèlement à l'objectivité propre au chercheur, le désenchantement ressenti par le pessimiste dont le fils risque sa vie au front. Par cette vibration du verbe, son argumentation nous invite à une méditation qui, au delà d'une réflexion psychanalytique, porte la marque subjective d'un aveu. Le traducteur ne peut que recourir à deux verbes différents « maintenir/endurer» et occulter l'effet de style freudien qui, par l'emploi du même radical verbal, visualise le processus de modification que Freud fait subir à l'adage en passant, par un jeu avec les particules verbales, du premier enjeu paix/guerre, au véritable enjeu: $\mathrm{vie} /$ mort. Le traducteur rend certes compte de cet écart qualitatif entre les deux couples d'opposés qui distinguent l'aménagement des relations entre États et l'aménagement de la relation de l'homme à son destin: il faut «maintenir» la paix, alors qu'il faut « endurer » la vie. Il ne peut cependant restituer le plaisir au jeu verbal qu'il partage avec l'écrivain dont il n'exprime, de ce fait, qu'imparfaitement la pensée puisque jeu et mélancolie y sont intimement mêlés.

\section{Traduction, dans la cure et l'écriture, d'une transmission traumatique hors langage}

Dans son ouvrage collectif pionnier de 1989, Violence d'État et psychanalyse, et notamment son article "Ruptures catastrophiques et travail de la mémoire », René Kaës rappelle que :

[C]e qui se transmet, dans la transsubjectivité des générations... c'est ce qui fait défaut, ce qui manque, ce qui n'a pas reçu d'inscription, ce dont l'inscription a été empêchée, ce qui a été nié, refoulé, ou forclos. (Kaës, 1989, 173)

Lors des relations infantiles précoces, une mère survivante n'est en effet pas en mesure de transmettre à son enfant une expérience dialectisable en mots sur ce qu'elle a vécu et sur la vision du monde qui lui en est restée. Alors que l'enfant s'approprie des savoirs sans saveur pour s'adapter au temps de la survie parentale, les paroles maternelles portent la saveur d'une secrète mélancolie mais ne profèrent à l'enfant aucun savoir transmissible, ou encore elles sont chargées de l'angoisse de nombreux affects incapables d'accéder à une secondarisation langagière. L'instance d'un ailleurs désirable parce qu'étranger à l'univers familier ayant été destituée sous l'emprise de la terreur, les parents survivants ne peuvent introduire leur enfant au monde des autres ni à celui de leurs mots. Cette bipartition dans la perception de l'environnement constitue ainsi chez leur enfant un clivage où l'expérience de deux espaces culturels en mutuelle exclusion réclame de lui une mutuelle traduction pour que s'effectue en lui un quelconque travail de subjectivation. 
Le traducteur qu'il devient peut alors éventuellement, au cours d'une analyse et dans l'après-coup libérateur de l'écriture, tenter de dénouer, dissoudre une charge d'angoisse pétrifiée autrefois au lieu même de l'ancienne impossibilité à parler. L'angoisse de jadis fait ainsi une dernière fois irruption par le travail de l'écriture avant de se dissiper, alors même que, dans l'enfance, l'absence de tout destinataire en mesure de recueillir la parole empêchait l'émergence d'un quelconque lieu d'énonciation.

Si je perds un lieu d'énonciation, écrit Todorov, je ne peux plus parler, je ne parle pas, donc je ne suis pas. (Todorov, 1985, 24)

Encore moins, est-on en droit de penser, si on n'a jamais pu occuper un tel lieu. L'écriture après coup n'a alors pour visée que celle de traduire une expérience archaïque en dévoilant violemment ce lieu d'énonciation d'un sujet en souffrance, devenu traducteur pour reprendre à son compte des affects épars, demeurés si longtemps en quête d'auteur.

La langue que je ne parlais pas autrefois, faute d'exister, je la parle aujourd'hui, mais en traducteur d'un lieu où je ne se trouve plus. Les stratifications du développement sont venues remanier, enrober d'un langage autre les affects infantiles restés sans nomination, si bien que c'est la double désinence du je parle à la première personne d'aujourd'hui et du je ne parlait pas à la troisième personne d'un autrefois imparfait qui se fait entendre en creusant simultanément les deux sillons du paradigme, en réduisant enfin le clivage entre le ressenti source et les moyens d'expression cible du traducteur, en réconciliant la voix toujours vivace de l'enfance et celle de l'analysant/écrivant, déterminé par son langage d'ici et de maintenant. L'expérience traumatique de base que ce type de bilinguisme s'épuise à répéter pour le réduire, est sans doute celle du bâillon qui empêchait de dire à la maison, aux premiers objets mutiques, l'écartèlement qui se vivait dehors, dans la langue de l'autre, puisque rien de ce dehors n'était présentifié, à l'écoute, dans l'espace de l'intimité. Mais pour celui qui cheminait ainsi d'un destinataire sourd à un autre, le dehors, l'école et ses institutrices ne pouvaient pas davantage recevoir, représenter les climats, les secrètes valeurs de la maison maintenue sous le sceau d'un non-lieu.

Chassé naguère de sa langue maternelle, comme ses parents le furent de leur espace de vie, un tel traducteur ne peut, par le métissage inhérent à son discours, que dérouter son destinataire, de même que son expérience personnelle lui a appris que comprendre l'autre, c'est d'une certaine façon s'expatrier. Il ne peut à présent que s'étonner de voir combien le prétendu monolingue (Derrida, 1996, 44) vit enfermé dans l'illusion de la communication, le déni d'un autre forclos dont, lui, occupe toujours clandestinement la place. C'est la contrainte de cette dissociation première que tentent inlassablement d'annuler les entrelacs de ses deux discours concomitants en dénonçant, à partir du lieu unique de son travail de traduction, les ruptures et les distorsions qui ont fracturé son histoire (Altounian, 1990, 147-150).

Une pratique du sens à traduire à un autre de langue étrangère ou une recherche du sens de l'héritage avec l'autre du transfert - en référence au tiers que représente 
soit le texte original soit le matériau originel - ne s'effectuent évidemment qu'avec l'acceptation d'une limite, c'est-à-dire le consentement à la perte d'un reste. J'ai pu écrire que traduire, c'était consentir à l'exil (Altounian, 2003, 92): s'il y a des similitudes entre l'expérience de l'exil et celle du transfert - dans le travail du traducteur, dans celui de l'analysant -, c'est parce que l'exilé doit non seulement faire le deuil du terreau de ses investissements initiaux, mais il doit encore, pour ne pas totalement les perdre, assumer la douleur tout aussi vive de réinvestir ces signifiants premiers - pour ainsi dire clandestinement en marrane - dans les mots et les valeurs de la langue d'accueil.

Lors des exils violents, ce qui dans un après-coup signe douloureusement le traumatisme de la rupture territoriale c'est, en effet, celui de la rupture culturelle et langagière. L'exilé doit traduire les traces de ses premiers référents existentiels en ceux qui devront désormais servir de cadre aux nouvelles modalités d'investissement de ses premiers objets. La mise en parallèle des deux processus de traduction s'impose d'autant plus, dans le cas présent, qu'il s'agit de traduire le fondateur d'une méthode d'investigation dont le dispositif, la cure, a précisément pour objet le «transfert», soit la transplantation des premiers liens affectifs dans le champ transférentiel et, grâce aux remaniements possibles dans cette nouvelle implantation, la résolution de certaines souffrances. N'a-t-on pas affaire avec Freud à un innovateur qui, héritier sans doute des nombreux exils de son ascendance persécutée, porte nécessairement dans son mode de penser et d'écrire, le modèle du dispositif d'un exil agi qu'il a créé?

Ainsi, traduire une absence de langue en une langue faisant advenir la langue absente à la parole, amener à l'écriture un passé traumatique infantile ou transgénérationnel qui, jusqu'alors, ne disposait pas de mots constitue une opération qui n'est pas sans rapport avec traduire d'une langue à une autre : dans chacune des deux postures, le traducteur est le seul à connaître la non-coïncidence des valences, soit entre deux systèmes de pensée, soit entre une pensée survivant à la mort psychique d'une partie de soi et celle restée indemne de cet éclatement. Si, selon Claude Janin, le trauma opère une "détransitionnalisation» (Janin, 1996, 23-24) entre la réalité psychique et la réalité factuelle, on peut avancer que toute traduction est une entreprise de transitionnalisation. Le travail de ce traducteur que l'héritier de survivants est devenu exhume en somme ce «non-traduit enclavé » (Laplanche, 2007, 201), que Jean Laplanche met en lumière dans sa théorisation de l'appareil psychique. Son écriture offre non seulement un linceul pour inhumer les morts laissés sans sépulture mais aussi une médiation, initialement absente, entre l'espace terrorisant des violences meurtrières et le monde susceptible de les écouter car, comme nous le rappelle René Kaës, « [1] e trauma est [...] le résultat d'une mise en échec de la formation intermédiaire » (Kaës, 1993, 54).

Dans les deux configurations ici convoquées, la préoccupation majeure est certes de sauver le plus possible de ce qui se perd nécessairement dans une mutation dont la castration inévitable conditionne néanmoins la possibilité de toute transmission. Il convient pourtant de remarquer que c'est le reste intraduisible, soit la récusation, opposée par un reste, à une transférabilité totale qui garantit la distance interposée entre la langue mélancolisante d'un monde éteint et celle qui permet une pensée 
vivante. C'est également ce reste qui inscrit la différence irréductible entre un mode de penser dans une langue et celui dans une autre langue et qui incite toute pulsion de vie à jouir de cette différence.

En revanche lorsqu'un traducteur occulte le lieu de la perte en ne percevant pas ce reste échappant à la traductibilité, il se comporte vis à vis du texte transmis comme un héritier qui effacerait la trace de ce qui n'a pu se dire dans son héritage, alors que c'est ce non-parlé qui a été pourtant fondateur de son existence. Il étouffe l'espace de subjectivité qui a produit la pensée et son écriture, occulte le lieu psychique qui a engendré le texte. Antoine Berman rappelle sans détours cette fonction éventuellement effractante de la traduction et la responsabilité du traducteur face à la parole de l'original dont il est l'unique transmetteur:

Seul le traducteur [...] peut percevoir ce qui, dans un texte, est de l'ordre du «renié », [...] Une traduction [...] a pouvoir de révéler ce qui, dans [une] œuvre est origine (inversement, elle a pouvoir de s'occulter elle-même cette possibilité), et cela indique qu'elle entretient avec elle un certain rapport de violence. Là où il y a révélation de quelque chose de caché, il y a violence. (Berman, 1984, 272)

S'il y a une interrelation entre traduction et transmission traumatique, constater l'écart du non-traduisible ne revient donc pas à l'effacer ni renier la butée féconde qu'il représente. C'est pourquoi je pourrais dire que la langue que m'apprit l'École de la République d'autrefois fut pour moi celle d'une première traduction, avec un reste évidemment intraduisible, traduction douloureusement mais heureusement castratrice qui me rendit ensuite possible, d'une part la traduction par l'écriture d'une transmission psychique et d'autre part le plaisir à la traduction de Freud.

\section{Inadéquation au langage inhérente à l'expérience des survivants}

Luba Jurgenson, spécialiste des témoignages sur les camps d'extermination nazis et soviétiques, distingue dans une étude remarquable, ce qu'elle appelle "le brouillon perdu ", c'est-à-dire les traces non verbalisées, non encore verbalisables de l'expérience immédiate chez le survivant, d'avec ce qui va devenir « le livre I ${ }^{4}$ », l'écrit que celui-ci sera à même de transcrire ultérieurement à partir de ce «brouillon». Concernant cette expérience qui ne peut encore se dire, elle écrit :

[L]a question qu'énonce ici Chalamov est celle de la traduction, du passage de la langue de l'expérience vers celle du texte. On constate l'inadéquation des deux langues: celle de l'expérience est infiniment pauvre, mais, aussi absolument complète, elle ne connaît pas de manque; la langue d'arrivée est riche au point de pouvoir dire sa propre insuffisance. L'expression du manque suppose, en effet, une grande complexité. (Jurgenson, 2003, 117)

On pourrait estimer que ce «brouillon perdu», pour le futur lecteur puisque en-deçà du langage, est semblable à l'expérience que les parents survivants ne peuvent 
et ne doivent pas restituer à leurs enfants qui, l'ayant inconsciemment perçue, ont, par leur acculturation au monde des «normalement vivants", quelque peu acquis cette «expression du manque» qui peut faire d'eux des transcripteurs de ce «brouillon perdu». Leur démarche en quelque sorte de sauvetage après coup de ce que recélait le silence des ascendants s'apparente à celle de ces traducteurs de textes anciens dont les originaux perdus ne nous sont parvenus que grâce à leur traduction dans une autre langue ${ }^{5}$.

Cette notion de «brouillon » va d'ailleurs exactement dans le sens de la remarque de Shoshana Felman sur la dépossession quant à la langue chez des parents survivants:

Une victime, par définition, ce n'est pas seulement quelqu'un qui est opprimé, mais aussi quelqu'un qui n'a pas de langage propre, quelqu'un qui s'est fait voler le langage dans lequel il aurait pu articuler sa victimisation. Le seul langage dont il dispose est celui de l'oppresseur, et dans le langage de l'oppresseur la victime, si elle se décrit comme victime, paraîtra folle même à ses propres oreilles. (Shoshana Felman, $1990,24)$

Une langue amputée de ses référents étrangers, disqualifiés par l'impunité des crimes vécus en l'absence de tout autre, est en effet comme une langue délirante. Comparons ici les réflexions de Rachel Ertel sur la mise à mort du yiddish à celles de Krikor Beledian sur la souffrance d'un écrivain arménien en proie à la destitution de sa langue disparue faute de territoire et d'assignataire. Tous deux évoquent de façon saisissante l'absence d'autre et donc d'espace de jeu métaphorique dans l'usage des langues aux locuteurs exterminés et aux cultures anéanties:

La parole yiddish circulait ésotérique, hermétique, entre rescapés et survivants d'un univers aboli, pour se réverbérer dans le vide. [...] Pour les rescapés, cette langue, seul vestige d'avant le déluge, n'est partageable qu'avec leurs pareils, elle ne saurait être transmise. [...] Si la langue semble faite pour ne pas être comprise ni même entendue, l'écriture en cette langue apparait, à ceux qui en sont les héritiers potentiels, comme le recours délibéré à une cryptographie ésotérique. L'accès en est barré pour toujours. Car derrière l'acte qui se veut acte d'initiation, de dévoilement, se cache l'acte de rétention et de voilement. (Ertel, 2001, 82-83)

Le texte [de Nartouni] [...] «Nostalgie de la langue arménienne» se constitue autour de la réflexion d'un collègue psychiatre d'origine grecque qui dit au narrateur «tu oublieras ta langue». Le narrateur qui n’a pas parlé dans sa langue maternelle depuis longtemps fait un rêve où il parle dans sa langue avec un chat, ensuite il s'en va dans les champs pour poursuivre son monologue « afin que personne n'entende mon délire et ne me prenne pour un fou » [...] Le narrateur restitue ici une expérience commune aux exilés: il porte en lui-même une langue qu'il ne peut pas partager, avec laquelle il ne peut échanger. Toute tentative de parler sa langue, dans 
la solitude de l'exil, fait de cette langue l'expression d'un délire et d'une folie. Est-ce un hasard si le narrateur se présente ici comme étant dans un asile de fous? Sa langue devient le signe de son enfermement et c'est pour cela que le collègue grec lui suggère l'oubli. (Beledian, 2001, 181-182) ${ }^{6}$

Or une telle pénurie en mots subjectivés/subjectivants, qui nous instituent parlant pour avoir été parlés, crée parfois chez l'enfant de survivants une grande appétence aux mots, à ceux du plaisir littéraire - plaisir exacerbé dans l'activité de traduction d'une pensée radicalement innovante comme celle de Freud - mais aussi à ceux qu'il va s'employer à rechercher dans la cure analytique afin de comprendre, en s'entendant prononcer des énoncés inédits repris par l'autre du transfert, ce que n'ont pu proférer ses ascendants; et ce, afin de pouvoir enfin les comprendre et les aimer sans empêchement.

\section{Portée politique de l'amour des mots traducteurs de l'héritage}

Les effets de subjectivation de cette relation d'amour après coup, dont sont alors investis les mots de la langue et de la culture, montrent d'ailleurs que seul cet amour peut soustraire un être pensant au pouvoir aliénant des langages totalitaires. C'est, par exemple, ce dont témoigne Sebastian Haffner en montrant dans son Histoire d'un Allemand (Haffner, 2002) que ce qui lui rendit impossible, à la différence de ses collègues, la moindre adhésion au national-socialisme et le contraignit à émigrer tenait à l'attachement inconditionnel qu'il avait, à la fois dans le respect et l'autonomie, pour la langue de culture transmise par son père.

On peut aussi déclarer, en référence aux témoignages de Victor Klemperer et de Charlotte Beradt sur la terreur des États exterminateurs, que si les totalitarismes visent à la «désolation » de ceux qui y survivent, il incombe à leurs héritiers de percevoir et de recueillir les quelques bribes, malgré tout psychisés, de ces plages de désolation autistique, d'accueillir ces ascendants en leur offrant leur propre altérité réceptrice de passeur et, ainsi traduits, de les transmettre au monde. Dans un des rêves collectés par Charlotte Beradt $(1966,41)$, une femme rêve qu'elle se parle en langue étrangère afin de ne pas se comprendre elle-même, de n'être comprise de personne, d'être ainsi assurée d'échapper à la censure et donc à la menace de mort. On pourrait comparer le parasitage douloureux de la langue et de l'écoute entre des survivants ayant connu la terreur et leurs enfants à celui de cette "langue étrangère » interposée entre ce rêveur, émetteur d'une langue incomprise de lui-même, et cet auditeur sous protection qu'il en devient.

Dans la postface de son livre $(1975,364)$, Victor Klemperer raconte qu'il hésitait à le publier lorsqu'il se souvint de l'explication d'une ouvrière sur son année passée en prison tandis que, de son coté, son mari communiste avait été détenu de nombreuses années. Elle lui donna la raison de son emprisonnement dans un savoureux patois berlinois : «wejen Ausdrücken », soit: en raison "des façons de parler» offensant le régime. Alors, en raison « des façons de parler» qui avaient pu emprisonner, pourchasser et assassiner, le philologue se décida à la publication. Les mots qui ont 
manqué, ceux qui ont été censurés ou dévitalisés par la démétaphorisation à l'œuvre dans les idéologies totalitaires et leurs meurtres sont à l'évidence des objets à sauver, des objets de recherche, de soins et d'amour.

À l'extrême opposé du langage idéologique qui évacue les ambiguïtés et les métaphores en se servant des mots pour supprimer la pensée et se substituer à elle, le rapport de Freud à la langue avec laquelle il pense et élabore sa recherche met en évidence les multiples innervations de sens qui traversent les mots. C'est sans doute ce qui lui inspire l'instauration d'une écoute analytique qui, à travers la vibration des mots, accède aux complexités du monde psychique et de la vie de l'esprit. Les énoncés mêmes de ses investigations, visualisent parfois ce qu'il est en train de théoriser; ce qui, évidemment, peut difficilement passer dans le texte traduit d'arrivée. Ses perlaborations sont ainsi souvent ponctuées des indices d'un inconscient chercheur qui jalonnent les chaînons par où chemine son enquête et qui, bien sûr, ne peuvent se percevoir que lors de la lecture de l'original. Le traducteur n'en devient conscient que par son impuissance à les faire passer dans l'autre langue. Tout comme pour l'héritier de mondes rendus muets, il ne lui reste que le plaisir et la douleur d'un reste impossible à traduire. En conséquence il serait totalement faux, voire éminemment injurieux à l'égard de Freud de prétendre que la langue allemande était seule appelée à faire surgir la psychanalyse: ce n'est pas de la langue allemande qu'est née la psychanalyse mais de ce rapport particulier que Freud entretient avec elle : il s'y révèle créatif et innovant, tout à la fois ludique et spéculatif, curieux des énigmes de la parole et imperturbable dans l'art de les déchiffrer, détecteur acharné des désirs qui s'y dissimulent et observateur non dénué d'humour de leur destin inassouvi.

\section{Conclusion: vertu analytique d'un atelier de traduction}

J'aimerais, pour conclure, évoquer ici un épisode pris sur le vif d'une séance de travail sur le texte freudien, car il représente à mes yeux une sorte de répétition anodine, dans le champ de la traduction, de ce qui relevait plus haut du champ de la transmission: au sein des équipes de traduction auxquelles je participe, ma pratique plus sensible à la langue source qu'à la langue cible, me fait revivre parfois un malaise que j'ai dû sans doute ressentir jadis lorsque, familière de deux codes de déchiffrement des choses de la vie, j'étais dans l'impuissance de signifier mon monde à moi dans celui de l'école. La répétition de cette impuissance cessa pourtant peu à peu de produire ses effets, lorsqu'il me fut donné de vivre ces moments miraculeux où, faisant écho à l'expression de mon mécontentement: «Ce n'est pas exactement ça ! », un cotraducteur, identifié probablement à mon insatisfaction, offrait le mot qui me manquait. Ce mot pertinent qui surgissait pour satisfaire au mieux les exigences du sens, ne ressemblait-il pas au mot de l'analyste qui, pendant la cure, nomme et pacifie le chaos d'affects où se débat son patient?

Le bonheur ressenti dans ces moments n'est pas tant celui de détenir enfin la solution recherchée mais il est de pouvoir partager avec autrui le même mouvement psychique de la pensée. L'aventure de cette trouvaille à plusieurs ne fait que témoigner d'une appartenance commune à la vie de l'esprit en affirmant et pré- 
sentifiant l'existence de celle-ci. Ce partage du plaisir à nommer vient modestement lier ce qui s'était rompu lors de l'écrasement culturel du monde de l'enfance. C'est bien cette " confiance dans les mots» (Chiantaretto, 2009) qui rend possible, dans le champ transférentiel, de faire parler des traces muettes et, dans un groupe de traduction, de sublimer en plaisir la capacité à percevoir et nommer ce qui se dérobe à soi ou à l'autre. C'est cette confiance dans les mots dont le juif traqué Klemperer tient à décrire, sous peine de mort, les multiples modalités d'effondrement. Quant aux parents survivants ils montrent, par leur usage d'un parler ressassant ou indigent, que cette confiance et ce plaisir ne sont qu'un luxe dont il leur a bien fallu se passer!

Janine Altounian

18, av. Gl. Leclerc, Paris 75014 janine.altounian@free.fr

\section{Notes}

1. «übersetzen» s'emploie plus fréquemment pour signifier: «traduire», mais Freud emploie également «übertragen» dans ce sens-là. Le terme en usage dans la traduction française est donc, pour le substantif correspondant: «Übertragung», «transfert» alors qu'il inclut aussi le sens de «traduction».

2. «La réflexion de Janine Altounian devient plus riche d'enseignement pour la pratique analytique de la textualité et pour sa saisie interprétative par les mots lorsqu'elle examine ce que la traduction fait apparaître, son gain et ce qu'elle ne peut manquer de perdre, son reste [...] L'interprétation privilégie les restes [...] Ce qui intéresse sa vigilance, c'est leur mobilité, leur circulation [...] Ce qu'[elle] privilégie rétroactivement comme son efficace c'est un inédit du reste, non sa fidèle répétition ». Observations faites dans le cadre des Tables rondes: L'impact des mots, organisées le 10 septembre 2005 au Quatrième Groupe autour de trois ouvrages: Janine Altounian (2003); Charlotte Beradt (1966); Victor Klemperer (1975).

3. «Zeitgemässes über Krieg und Tod»: «Wir erinnern uns des alten Spruches: Si vis pacem, para bellum. Wenn du den Frieden erhalten willst, so rüste zum Kriege. Es wäre zeitgemäß, ihn abzuändern: Si vis vitam, para mortem. Wenn du das Leben aushalten willst, richte dich auf den Tod ein"

4. Elle désigne également « livre II » l'ouvrage où l'auteur réfléchit ensuite sur l'expérience qu'il a transcrite dans le «livre I». Ainsi au Livre I de Primo Levi, Se questo è un uomo, 1947, correspond le Livre II, I sommersi e i salvati, 1986.

5. Ainsi par ex. les Chroniques d'Eusèbe de Césarée, dont les originaux furent écrits par des auteurs non-arméniens et qui ne nous sont parvenus que par leur traduction en langue arménienne.

6. Nartouni est l'un des écrivains de langue arménienne des années trente, exilés en France dont traite cet ouvrage de Beledian.

\section{Références}

ALTOUNIAN, J., 1990, Ouvrez-moi seulement les chemins d'Arménie, Un génocide aux déserts de l'inconscient (Préface de René Kaës), Les Belles Lettres/Confluents psychanalytiques, 1990, 2003 (2éd.). dont «Faute de parler ma langue».

ALTOUNIAN, J., 2000, 2003, La Survivance/Traduire le trauma collectif (Préface de Pierre Fédida, Postface de René Kaës), Dunod/Inconscient et Culture.

ALTOUNIAN, J., 2003, L'écriture de Freud. Traversée traumatique et traduction, PUF/bibliothèque de psychanalyse.

ALTOUNIAN, J., 2005, L'intraduisible/Deuil, mémoire, transmission, Dunod/Psychismes. 
ALTOUNIAN, J., 2009, Mémoires du Génocide arménien. Héritage traumatique et travail analytique de Vahram et Janine Altounian, avec la contribution de K. Beledian, J. F. Chiantaretto, M. Fraire, Y. Gampel, R. Kaës, R. Waintrater, PUF.

BELEDIAN, K., 2001, Cinquante ans de littérature arménienne en France; Du même à l'autre, CNRS Éditions, Paris.

BERADT, C., 1966, Das dritte Reich des Traums, Suhrkamp Taschenbuch, traduit par Pierre SaintGermain: Rêver sous le Troisième Reich, Payot et Rivages, 2002.

BERMAN, A., 1984, L'épreuve de l'étranger, Gallimard, 1984. (notamment le chapitre: «Hölderlin: le national et l'étranger »)

CHIANTARETTO, J.-F., 2009, Naissance d'un témoignage, témoignage d'une naissance, Mémoires du Génocide arménien. Héritage traumatique et travail analytique de Vahram et Janine Altounian, avec la contribution de K. Beledian, J. F. Chiantaretto, M. Fraire, Y. Gampel, R. Kaës, R. Waintrater, PUF, 2009.

DERRIDA, J., 1996, Le monolinguisme de l'autre, Galilée.

ERTEL, R., 2001, Le Yiddish : La langue et la crypte, Les Temps modernes, 615-616.

FELMAN, S., 1990, À l'âge du témoignage: Shoah de Claude Lanzmann, Au sujet de Shoah, le film de Claude Lanzmann, Belin.

FREUD, S., 1915, Zeitgemässes über Krieg und Tod, in GW II/III, 1915; traduction: Actuelles sur la guerre et la mort, in OCF/P XIII, 1988.

HAFFNER, S., 2002, Histoire d'un Allemand, Souvenirs (1914-1933), Actes Sud, traduit de l'allemand par B. Hébert.

JANIN, C., 1996, Figures et destins du traumatisme, PUF.

JURGENSON, L., 2003, L'expérience concentrationnaire est-elle indicible? Éditions du Rocher, 2003.

KAËS, R., 1989, Ruptures catastrophiques et travail de la mémoire », in Violence d'État et psychanalyse, Dunod.

KAËS, R., 1993, Le sujet de l'héritage, in Transmission de la vie psychique entre générations, Dunod.

KLEMPERER, V., 1996, L.T.I., Notizbuch eines Philologen, Reclam, Leipzig, 1975, traduit par Élisabeth Guillot: L.T.I., La langue du III ${ }^{e}$ Reich, Paris, Albin Michel, 1996.

LAPLANCHE, J., 2007, Sexual, La sexualité élargie au sens freudien, 2000-2006, PUF.

TODOROV, T., 1985, Bilinguisme, dialogisme et schizophrénie, in Du bilinguisme, Denoël.

ZALTZMAN, N., 2006, L'impact des mots, Topique 96, août 2006. 